\title{
Specificity versus detectable polymorphism in host-parasite genetics
}

\author{
STEVEN A. FRANK
}

Department of Ecology and Evolutionary Biology, University of California, Irvine, California 92717, U.S.A.

\section{SUMMARY}

Detectable polymorphism of host resistance and parasite host range is a poor guide to the specificity of a host-parasite interaction. I analyse a simple haploid model to support this claim. The model assumes that the true specificity is 'matching-allele', in which each of $n$ host alleles causes resistance to only one of $n$ different parasite alleles. The detectable polymorphism in samples from a matching-allele system would lead one to infer the gene-for-gene specificity commonly observed in plant-pathogen interactions. Gene-for-gene and matching-allele specificity require very different fitness assumptions to explain observed patterns of polymorphism. Yet models for each type of specificity can easily be constructed that fit the available data. In addition, the currently favoured 'elicitor-receptor' model for the biochemistry of plant-pathogen recognition agrees equally well with gene-for-gene or matching-allele specificity. I do not claim that the simple matching-allele specificity is the correct model for plant-pathogen genetics. My point is that one cannot reconstruct both specificity and population history from patterns of resistance among host-parasite pairs in a sample. I draw two conclusions: first, inferred specificity and polymorphism are only useful when compared with a family of theoretical models; and secondly, biochemical models of specificity must be tested by their population genetic consequences.

\section{INTRODUGTION}

The goal of population genetic analysis is to explain the forces that influence polymorphism. There is, however, a serious problem in the genetic analysis of hostparasite systems because the definitions of genotypes are confounded with the polymorphism that one seeks to explain. For example, a group of hosts may be clustered as a single genotype because each host responds in the same way to all available parasites. A new sample of parasites may separate this cluster of hosts into different response groups. Thus any inference about host-parasite specificity depends on detectable polymorphism.

My claim is that, by using polymorphism to define the problem, one loses the ability to explain polymorphism. To break the loop one needs an extrinsic method to define specificity, such as the biochemistry of host-parasite recognition.

To illustrate the difficulty with coevolutionary systems, here are the steps that have been used to study polymorphism in cases such as plant-pathogen genetics (Burdon 1987). First, a model for the specificity of host-parasite genetics is developed. This model is based on the phenotypic interactions between samples of hosts and parasites and the mendelian segregation ratios in crosses. Secondly, the model of specificity is used to infer the frequency of host resistance and parasite host-range genotypes. Thirdly, population genetic models, based on the inferred genetic specificity, are developed to explain the observed genotypic frequencies.
I analyse the gene-for-gene interaction between plants and pathogens (Flor 1956, 1971) to show that this procedure may be misleading. I show that both the inferred gene-for-gene specificity and the associated explanation for the population frequencies of resistance and susceptibility are consistent with a different model of specificity. My alternative model for plant-pathogen specificity, which I call the 'matching-allele' model, is based on the currently favoured elicitor-receptor model for the biochemistry of plant-pathogen recognition (Gabriel \& Rolfe 1990; Keen \& Dawson 1992). My analysis shows that different assumptions about the true specificity of a host-parasite system may yield similar inferences about specificity and detectable polymorphism.

\section{SPECIFICITY}

I analyse a model with a single haploid locus. Each of the $n$ host alleles causes recognition and resistance to only one of the $n$ parasite alleles. Thus each host is resistant to $1 / n$ of the parasite genotypes, and each parasite can attack $(n-1) / n$ of the host genotypes (Frank 1991). I call this the 'matching-allele' model.

The patterns of resistance and virulence in the simple $2 \times 2$ case of the matching-allele model are distinct from the classical gene-for-gene interaction of plants and pathogens. Figure $1 a$ shows the standard gene-for-gene model (Flor 1956, 1971; Burdon 1987). The host has two phenotypes, resistant $(R)$ and susceptible $(S)$. The pathogen (parasite) has two 
(a)

\begin{tabular}{c|c|c|}
\multicolumn{1}{c}{} & \multicolumn{1}{c}{$A$} & \multicolumn{1}{c}{$V$} \\
\cline { 2 - 3 }$S$ & - & + \\
\cline { 2 - 3 }$S$ & + & + \\
\cline { 2 - 3 } & & \\
\cline { 2 - 3 } & &
\end{tabular}

Figure 1. Resistance $(-)$ and susceptibility $(+)$ between two host alleles and two parasite alleles. (a) Gene-for-gene specificity. (b) Matching-allele specificity.

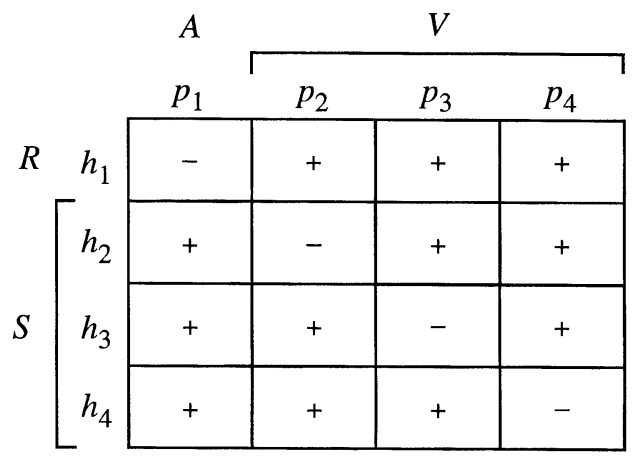

Figure 2. Resistance $(-)$ and susceptibility $(+)$ in a matching-allele model with four alleles.

phenotypes, avirulent $(A)$ and virulent $(V)$. Plant pathologists use the term 'virulence' for host range. I follow their convention.

In the gene-for-gene model, a host resists attack only when the host-parasite pair has an $R: A$ match. Person \& Mayo (1974) refer to this match as a 'stop-signal'. Recent biochemical models suggest that the avirulence allele $(A)$ produces a gene product (elicitor) that can be recognized only by specific host receptors $(R)$. This specific elicitor-receptor recognition induces a nonspecific set of host defence mechanisms (Gabriele \& Rolfe 1990).

Figure $1 b$ shows the susceptibility and resistance patterns for the matching-allele model with $n=2$. In this model each parasite genotype functions as either an avirulence allele or a virulence allele depending on the host genotype. By contrast, the gene-for-gene system always has a universal virulence allele that can attack all host genotypes. Similarly, each host genotype in the matching-allele model functions as either a resistance or a susceptibility allele depending on the parasite genotype. The classical gene-for-gene system always has a universal susceptible genotype that can be attacked by all parasite genotypes.

I chose to study matching-allele specificity because it is consistent with the currently favoured elicitorreceptor model for the biochemical basis of recognition (Gabriel \& Rolfe 1990; Keen \& Dawson 1992). In the simple version of the matching-allele model, there is a one-to-one correspondence between elicitors and receptors. In the gene-for-gene model the universal virulence allele does not produce an elicitor that can be recognized by any of the available host receptors. Similarly, the universal resistance allele does not produce a receptor that can recognize any of the available pathogen elicitors (see Discussion).
I show that the matching-allele model leads to inferred patterns of resistance and virulence that appear much more like the classical gene-for-gene specificity (figure $1 a$ ) than the matching-allele specificity (figures $1 b$ and 2). I use the word 'inferred' to mean the specificity of the host-parasite interaction that appears to fit the data given the detectable polymorphism in the system.

\section{DYNAMICS OF THE MATCHING-ALLELE MODEL}

I use a system of Lotka-Volterra equations for the dynamics of the matching-allele model (see, for example, May 1974). These equations describe the dynamics of genotype abundances rather than just the relative genotype frequencies. Thus the model tracks epidemic fluctuations in population sizes and disease intensity in addition to changes in genotype frequency. The model is:

$$
\begin{aligned}
& \Delta h_{i}=h_{i}\left[r-H / K-m\left(P-p_{i}\right)\right] \Delta t, \\
& \Delta p_{j}=p_{j}\left[-s+b\left(H-h_{j}\right)\right] \Delta t .
\end{aligned}
$$

The values of $h_{i}$ and $p_{j}$ are the abundances of hosts of genotype $i$ and parasites of genotype $j$. The total abundance of hosts is $H=\sum_{k=1}^{n} h_{k}$, and the total abundance of parasites is $P=\sum_{k=1}^{n} p_{k}$.

The term $r$ is the host's intrinsic rate of increase; $H / K$ is the strength of density-dependent competition among hosts with carrying capacity of $K ; m$ is the morbidity and mortality per parasite attack; $s$ is the parasite death rate; and $b$ is the parasite's intrinsic birth rate per host-parasite contact. The $\Delta t$ term is the size of the time step over which the interactions occur. For example, $\Delta t$ may be the length of one host generation or one season in a discrete-time model. When birth, death and disease cause continuous change of the abundances of hosts and parasites, $\Delta t \rightarrow 0$.

The system in equation (1) is easier to analyse when rewritten in non-dimensional form (Segel 1972; Murray 1989). Non-dimensional analysis focuses attention on a minimal set of parameters and highlights relative magnitudes (scaling relations) among the processes that drive the dynamics. This is accomplished without altering the dynamics or interpretation because one can translate freely between the biologically motivated formulation and the non-dimensional quantities.

The system can be rewritten with the following substitutions:

$$
\begin{aligned}
\hat{h}_{i} & =h_{i} / K, \quad \hat{p}_{j}=m p_{j} / r, \quad \tau=r \Delta t, \\
\hat{s} & =s / r, \quad \hat{b}=K b / r .
\end{aligned}
$$

Omitting the hats yields the non-dimensional system

$$
\begin{aligned}
\Delta h_{i} & =h_{i}\left[1-H-\left(P-p_{i}\right)\right] \tau, \\
\Delta p_{j} & =p_{j}\left[-s+b\left(H-h_{j}\right)\right] \tau .
\end{aligned}
$$

I present the equilibria and stability analysis in Appendix 1 . The dynamics of the system are controlled by the equilibrium with all hosts and parasites 
random immigration into the system cause unpredictable fluctuations in the composition of the four host and parasite genotypes (figure 5).

\section{SPEGIFICITY AGAINST DETECTABLE POLYMORPHISM}

Suppose that a host-parasite system interacts according to the matching-allele model with $n$ alleles. What type of genetic system and pattern of polymorphism would one infer from samples of the host and parasite populations? The standard procedure is to isolate some host and parasite lines and then test each host against each parasite for resistance or susceptibility.

Here is a reasonable method of classification: (i) find the host genotype that resists the highest proportion of parasites in the sample; label that host genotype $R$ for resistant; only those hosts that resist exactly the same set of parasites are classified as $R$; (ii) label all other hosts as $S$ for susceptible; (iii) label all parasites that cannot attack host genotype $R$ as $A$ for avirulent; and (iv) label all other parasites as $V$ for virulent.

After following this procedure, one has a classification that matches the gene-for-gene system in figure $1 a$. What is the frequency of host-parasite pairs that would be misclassified if the true system were a matching-allele model with $n$ alleles? The $R$ and $A$ alleles were defined strictly by their response in the sample, so there can be no errors in any host-parasite pair in which the host is classified as $R$ or the parasite as $A$. All errors must occur when a host-parasite pair, classified as $S: V$, yields a resistant reaction rather than the predicted susceptible response.

The gene-for-gene pattern in figure $1 a$ would typically provide a good fit to the observed patterns of resistance and susceptibility. To support this claim, I analyse the matching-allele system in two steps. First, I derive some simple analytical conclusions for the system when at its equilibrium with all allelic types equally frequent. Secondly, I use computer simulations across a range of parameter values to summarize the fit between the gene-for-gene predictions and the inferred genetic system.

\section{ANALYTIG CONGLUSIONS AT EQUILIBRIUM}

Consider, for example, what would be observed if the true genetic system followed the matching-allele pattern in figure 2 with $n=4$. If the system were at equilibrium, then each of the $n$ host and parasite genotypes would be equally frequent. The $n^{2}$ hostparasite pairs would also be equally frequent. This case, with uniform frequency for each cell in an interaction table, would yield samples that provided the maximum amount of information about the underlying specificity.

Figure 6 shows the proportion of each classification that would be inferred when the inference scheme listed above is applied to an equilibrium matchingallele system with $n$ alleles. The values are the number of cells from an $n \times n$ matching-allele interaction that
A

V

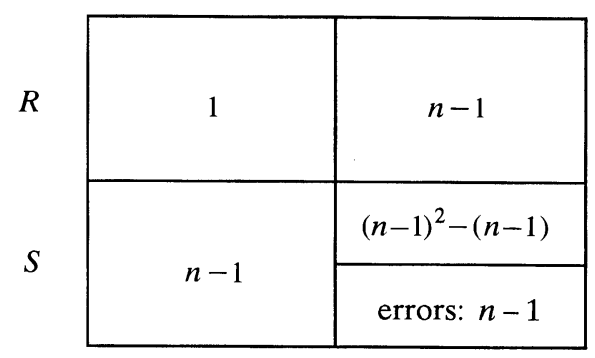

Figure 6. The proportions of each gene-for-gene classification that would be inferred from an equilibrium matching-alleles system with $n$ alleles. Frequencies are obtained by dividing by $n^{2}$. Details are presented in the text.

would be classified according to the four gene-for-gene pairs. Frequencies are obtained by dividing the number of cells by $n^{2}$.

The values in each of the four cells of figure 6 can be derived by examining figure 2. The choice for $R$ and $A$ alleles is arbitrary because all cells in figure 2 are equally frequent. Let $h_{1} \equiv R$ and $p_{1} \equiv A$. Thus the $R: A$ interaction occurs in one cell, a frequency of $1 / n^{2}$. The frequency of the $R$ and $A$ alleles is $1 / n$. The frequency of the $S$ and $V$ alleles is inferred to be $(n-1) / n$ because $S$ is defined as not $R$, and $V$ is defined as not $A$.

The rest of the values in figure 6 can now be filled in. The $S: A$ interaction corresponds to the first column of figure 2 excluding the first cell $\left(h_{1}: p_{1}\right)$, so there are $n-1$ cells for this interaction. Similarly, $R: V$ corresponds to the first row of figure 2, so there are also $n-1$ cells for this interaction. That leaves the submatrix of $(n-1)^{2}$ cells, excluding the first row and column, for the inferred $S: V$ interaction. In the gene-for-gene model of figure $1 a$, these are all susceptible $(+)$ interactions. From figure 2 one can see that the $n-1$ diagonal elements will be resistant (-) reactions, and the remaining $(n-1)^{2}-(n-1)$ will be of the predicted susceptible type. Thus the frequency of pairwise interactions incorrectly classified by the gene-for-gene prediction within the $S: V$ cells is $(n-1) /$ $(n-1)^{2}=1 /(n-1)$, and the frequency of misclassifications within the entire table is $(n-1) / n^{2}$. Paradoxically, the data fit the gene-for-gene predictions more closely as the number of alleles, $n$, increases.

\section{DETECTABLE POLYMORPHISM IN SIMULATED DATA}

In the previous section I drew conclusions about detectable polymorphism in the matching-allele model. Those conclusions depended on the assumption that the system is near its equilibrium. The analysis presented earlier showed, however, that the system is not stable for any parameter values. What is the error rate in the $S: V$ cell when allele frequencies fluctuate according to the dynamics of equation (3)?

I iterated equation (3) and repeatedly applied the inference method outlined above. This inference method classifies each host-parasite pair into one of the four cells shown in figure 6 . In addition, I divided the interactions in the $S: V$ cell into those that matched the 

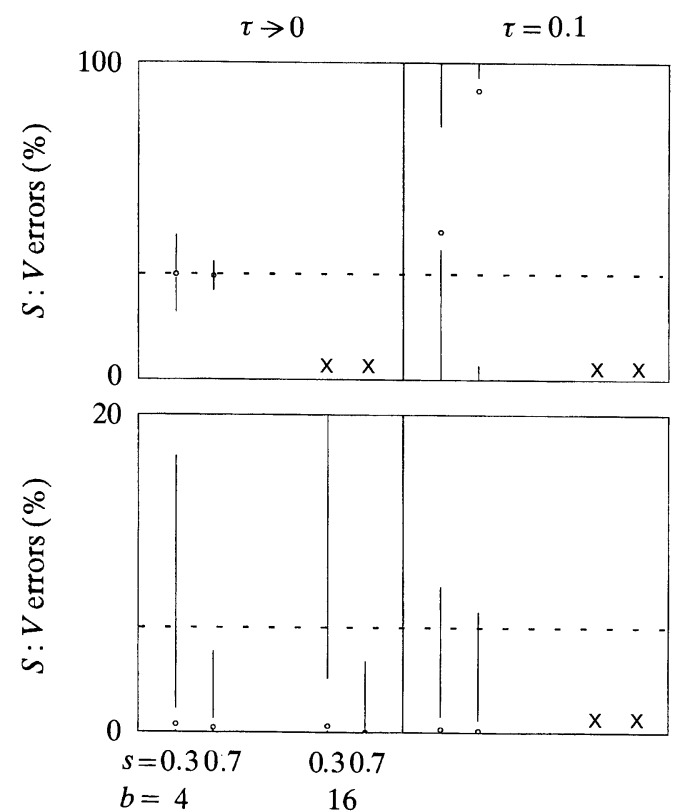

Figure 7. The percentage of errors in the $S: V$ cell of the genefor-gene classification when applied to a matching-allele system. The lower-left panel shows the arrangement for the four parameter combinations of $s$ and $b$. Extinction is simulated by setting to zero any abundance less than $10^{-4}$. Colonization is simulated by adding $10^{-4}$ to the abundance of each host and parasite, with 100 time units as the average period between colonization events for each type. Top, $n=4$; bottom, $n=16$.

prediction of susceptibility and those that had a resistant reaction. I refer to the frequency of resistant reactions within the $S: V$ cell as the frequency of errors.

In each run I calculated the frequency of errors at each of 200 equally spaced time points over a period of 2000 time units. An observation was discarded because of insufficient polymorphism if any of the frequencies of the four cells in figure 6 was less than 0.001 . I then calculated the 5th, 25th, 50th, 75th and 95th percentiles of the frequency of errors for the remaining observations. I repeated this procedure for different values of the four parameters, $s, b, n$ and $\tau$.

Figure 7 shows the results. Within each panel, the vertical lines and circles show the distributions of errors for particular parameter combinations. The circle is the median (50th percentile). The top of a line above a circle is the 95th percentile, the bottom of that line is the 75 th percentile. The top of the line below the circle is the 25 th percentile, the bottom of that line is the 5 th percentile. A cross shows a parameter combination for which $90 \%$ or more of the observations were discarded because of insufficient polymorphism.

The broken line shows the predicted frequency of errors at equilibrium according to the $1 /(n-1)$ rule. For $n=16$, more than $75 \%$ of the observations have a percentage of error in the $S: V$ cell of less than 6.7 $[1 /(n-1)]$. For $n=4$ and relatively high values of $b$, epidemics are severe and cause frequent local extinctions and little polymorphism. For relatively low values of $b$, the system is nearly stable when host-parasite interactions occur continuously in time $(\tau \rightarrow 0)$, but fluctuates widely when interactions occur in discrete time steps $(\tau=0.1)$

\section{DISGUSSION}

Detectable polymorphism is a poor guide to the specificity of a host-parasite interaction. I analysed one example to support this claim. If the true specificity were matching-allele with several allelic variants, then the detectable polymorphism would lead one to infer a gene-for-gene specificity (figure 7).

Several theoretical models have been developed to explain observed polymorphism in systems with apparent gene-for-gene specificity (reviewed by Leonard \& Czochor 1980; Levin 1983; Burdon 1987; Frank 1992, 1993). These models assume that virulence alleles have a negative effect on fitness that offsets the benefit of wider host range. This assumption is necessary because, without a fitness cost, the virulence allele would spread to fixation (in figure $l a, V$ has an advantage over $A$ ). A model that predicted fixation of virulence could not explain the observed polymorphism of virulence and avirulence alleles (Vanderplank 1968). A similar argument leads to the conclusion that resistance must have a cost.

The argument for costs can be summarized as follows. Detectable polymorphism suggests gene-forgene specificity. Given gene-for-gene specificity, there must be costs of resistance and virulence to explain the observed polymorphism. Models that assume costs predict that resistance alleles will be rare relative to susceptibility alleles, and virulence alleles will be common relative to avirulence alleles. Observations appear to support the predicted frequencies of these allelic classes (Frank 1992, 1993).

The matching-allele model also fits the available data: apparent gene-for-gene specificity; rare resistance and common virulence when one uses detectable polymorphism to infer specificity (figure 2); and a pattern of specificity that matches the currently favoured elicitor-receptor model for the biochemistry of plant-pathogen recognition (see the Specificity section). Note that the matching-allele model does not require costs of resistance and virulence to explain the data.

I do not claim that the simple matching-allele specificity is the correct model for plant-pathogen genetics. My point is that one cannot start with samples of hosts and parasites, test for resistance and virulence within the sample, and then use population frequencies and mendelian segregation ratios to reconstruct both specificity and population history. Barrett (1985) made a similar point about data from agricultural epidemics and plant breeding.

What can be done? A family of models can be developed that matches both detectable polymorphism and observations on the biochemistry of specificity. Population genetic models must predict detectable polymorphism and make reasonable assumptions about specificity. Biochemical models of specificity must be consistent with the inferred patterns of specificity and have population genetic consequences that match detectable polymorphism.

For example, a $2 \times 2$ case of the matching-allele model cannot be the true model of specificity for plant-pathogen systems because it does not yield 


\section{S. A. Frank Host-parasite genetics}

observations that look like the gene-for-gene system (figure 1 ). By contrast, a $16 \times 16$ case of the matchingallele model may be true because its detectable polymorphism would appear to have gene-for-gene specificity (figure 7).

I do not have space here to develop a family of models for plant-pathogen genetics: I limit myself to one extension of the matching-allele model. In that model, each pathogen allele makes a gene product (elicitor) that can be recognized by the product (receptor) of one host allele, the matching leading to a resistant reaction. A pathogen allele that made no product (a null allele) would therefore be universally virulent. Some mutations to virulence appear to be deletions (Flor 1971). Thus an expanded matchingallele model could include null pathogen alleles that are universally virulent. As noted above, universally virulent alleles would spread to fixation without an opposing cost. Null alleles must therefore have a fitness cost when they occur polymorphically with avirulence alleles that make recognizable gene products.

Alleles classified as virulence may be universally virulent because they are null, or they may produce gene products for which the sample of hosts does not have the associated recognition receptor. Pathogen alleles are sometimes classified as virulence on one host species although they function as avirulence alleles on a second host species (Gabriel \& Rolfe 1990). Such alleles probably produce specific gene products. Thus virulence may encompass both null alleles and alleles that produce potentially recognizable products.

When selection on the pathogen population is extreme, such as in epidemic situations or when screening for mutants in a laboratory, null virulence alleles are probably common because the selection intensity can overcome any non-lethal fitness cost of deletion or loss-of-function mutations. Allelic variants that produce slightly different gene products may be more common under less extreme situations, in which pathogens escape hosts through diversity over time or space (figure 5). In any case, the analysis of plantpathogen systems must continue back and forth between biochemical specificity and population genetic consequences.

The need for joint studies of specificity and polymorphism apply to other coevolutionary systems. For example, I studied the coevolutionary conflict between cytoplasmic and nuclear genes that determine male sterility in plants. I showed that the cytonuclear specificity and number of allelic variants cannot be inferred by analysing mendelian segregation ratios from samples of natural populations (Frank 1989).

My study of male sterility was more detailed than the matching-allele model here. In my male sterility analysis I inferred specificity from the mendelian segregation ratios of multilocus diploid genotypes. The conclusions were, however, the same. Specificity does not reveal itself through interaction matrices and segregation ratios.

My conclusions about plant disease and cytoplasmic male sterility contrast sharply with the common claim that detectable polymorphism must form the basis for the genetic assumptions of theoretical models. The truth is that detectable polymorphism cannot be understood without theories that are derived from other considerations.

I thank R. M. Bush for helpful comments on the manuscript, and S. A. Levin for teaching me the direct-product stability analysis used in the Appendix. My research is supported by NSF grant BSR-9057331 and NIH grants GM42403 and BRSG-S07-RR07008.

\section{APPENDIX 1}

In this Appendix I provide some analytical details about the equilibria and stability of equation (3). The system has $2^{2 n}$ subsystems classified by the presence or absence from the system of each of the $h_{i}$ and $p_{j}$, with $i, j=1, \ldots, n$. Only two classes of subsystem need to be considered because of the symmetry in host-parasite interactions and the fact that a host can resist only a matching parasite type. I assume $(n-1) b-n s>0$, otherwise parasites cannot be sustained.

The first class of subsystem has $0<k \leqslant n$ host types present, and $0 \leqslant r \leqslant n-k$ non-matching parasites. All matching parasites would be lost from this type of system because of host resistance. There are $\sum_{k=1}^{n}\left(\begin{array}{c}n \\ k\end{array}\right) \sum_{r=0}^{n-k}\left(\begin{array}{c}n-k \\ r\end{array}\right)=$ $3^{n}-2^{n}$ such systems. A rare host can invade the equilibrium of these systems if it matches one of the parasites that are present. In the case where there are $n$ hosts and 0 parasites, a rare parasite can invade (increase when rare) if $(n-1) b-n s>0$. Thus these subsystems are always unstable when there is the potential for parasitism.

The second class of subsystem has a matching host type present for each parasite type with positive abundance. There are $2^{n}$ such systems. The system in which there are no hosts or parasites is unstable because hosts can invade. When there are less than $n$ matching pairs, all unmatched hosts would be lost from the system because they have no benefits of resistance. The parasites can be sustained in a system with $k$ matching pairs only if $(k-1) b-k s>0$. A rare parasite can invade the equilibrium of these systems because it avoids matching host resistance. Thus, when there are less than $n$ matching pairs, these systems are always unstable when there is the potential for parasitism.

From these two classes of subsystem it can be seen that, in systems that can sustain parasitism, the equilibrium of interest is the case with $n$ matching host and parasite pairs. With all hosts and parasites present, the symmetry of the system implies that the equilibrium abundances of all host types are equal, $h_{i}^{*}=h^{*}$, and the equilibrium abundances of all parasite types are equal, $p_{j}^{*}=p^{*}$. This equilibrium occurs at $h^{*}=s /[b(n-1)]$ and $p^{*}=\left(1-H^{*}\right) /(n-1)$, where $H^{*}=n h^{*}$.

The stability of this equilibrium with $2 n$ dimensions can be analysed by an elegant (and apparently little-known) theorem of Friedman (1956) (see Othmer \& Scriven 1971; Levin 1974).

The Jacobian matrix of equation (3) can be written compactly as:

$$
(\boldsymbol{A}+\boldsymbol{B}) \otimes \boldsymbol{J}_{n}-\boldsymbol{A} \otimes \boldsymbol{I}_{n},
$$

where $\otimes$ is the direct product, also known as the Kronecker or tensor product (see, for example, Bellman 1960), $\boldsymbol{J}_{n}$ is a square matrix of size $n$ with ones in all positions, $\boldsymbol{I}_{n}$ is the identity matrix of dimension $n$, and

$$
\boldsymbol{A}=\tau\left(\begin{array}{cc}
0 & -h^{*} \\
b p^{*} & 0
\end{array}\right), \quad \boldsymbol{B}=\tau\left(\begin{array}{rr}
-h^{*} & 0 \\
0 & 0
\end{array}\right) .
$$

The matrices $\boldsymbol{I}_{n}$ and $\boldsymbol{J}_{n}$ are self adjoint and have common spectral representation. The eigenvalues of $\boldsymbol{I}_{n}$ are 1 (repeated 
$n$ times), and the eigenvalues of $\boldsymbol{J}_{n}$ are 0 (repeated $n-1$ times) and $n$. Thus, by Friedman's (1956) theorem, the eigenvalues of $(\boldsymbol{A}+\boldsymbol{B}) \otimes J_{n}-\boldsymbol{A} \otimes \boldsymbol{I}_{n}$ are simply the eigenvalues of $-\boldsymbol{A}$ (repeated $n-1$ times), given by the characteristic equation $\lambda^{2}+b p^{*} h^{*} \tau^{2}=0$, and the eigenvalues of $(n-1) \boldsymbol{A}+n \boldsymbol{B}$, given by the characteristic equation $\lambda^{2}+H^{*} \lambda \tau+b p^{*} h^{*}(n-1)^{2} \tau^{2}=0$. Stability is determined by the first characteristic equation. For continuous time, $\tau \rightarrow 0$, the $\tau$ s are dropped from the characteristic equations, the dominant eigenvalue has a real part of zero, and the system is neutrally stable. In discrete time the system is unstable when the dominant eigenvalue has a modulus greater than one, which occurs when $b p^{*} h^{*} \tau^{2}>0$. This condition is always satisfied at the internal equilibrium with all host and parasite types present, hence the system is always unstable when there are discrete time lags in the competitive effects among hosts and in the interactions between host and parasite.

\section{REFERENCES}

Barrett, J. A. 1985 The gene-for-gene hypothesis: parable or paradigm. In Ecology and genetics of host-parasite interactions (ed. D. Rollinson \& R. M. Anderson), pp. 215-225. New York: Academic Press.

Bellman, R. 1960 Introduction to matrix analysis. New York: McGraw-Hill.

Burdon, J.J. 1987 Diseases and plant population biology. Cambridge University Press.

Flor, H. H. 1956 The complementary genic systems in flax and flax rust. Adv. Genet. 8, 29-54.

Flor, H. H. 1971 Current status of the gene-for-gene concept. A. Rev. Phytopathol. 9, 275-296.

Frank, S. A. 1989 The evolutionary dynamics of cytoplasmic male sterility. Am. Nat. 133, 345-376.

Frank, S. A. 1991 Ecological and genetic models of host-pathogen coevolution. Heredity 67, 73-83.
Frank, S. A. 1992 Models of plant-pathogen coevolution. Trends Genet. 8, 213-219.

Frank, S. A. 1993 Coevolutionary genetics of plants and pathogens. Evol. Ecol. 7, 45-75.

Friedman, B. 1956 An abstract formulation of the method of separation of variables. In Proceedings of the conference on differential equations (ed. J. B. Diaz \& L. E. Payne), pp. 209-226. College Park, Maryland: University of Maryland Bookstore.

Gabriel, D. W. \& Rolfe, B. G. 1990 Working models of specific recognition in plant-microbe interactions. A. Rev. Phytopathol. 28, 365-391.

Keen, N. T. \& Dawson, W. O. 1992 Pathogen avirulence genes and elicitors of plant defense. In Genes involved in plant defense (ed. T. Boller \& F. Meins), pp. 85-114. New York: Springer-Verlag.

Leonard, K. J. \& Czochor, R.J. 1980 Theory of genetic interactions among populations of plants and their pathogens. A. Rev. Phytopathol. 18, 237-258.

Levin, S. A. 1974 Dispersion and population interactions. Am. Nat. 108, 207-228.

Levin, S. A. 1983 Some approaches to the modelling of coevolutionary interactions. In Coevolution (ed. M. H. Nitecki), pp. 21-65. University of Chicago Press.

May, R. M. 1974 Stability and complexity in model ecosystems, 2nd edn. Princeton University Press.

Murray, J. D. 1989 Mathematical biology. New York: Springer-Verlag.

Othmer, H. G. \& Scriven, L. E. 1971 Instability and dynamic pattern in cellular networks. J. theor. Biol. 32, 507-537.

Person, C. \& Mayo, G. M. E. 1974 Genetic limitations on models of specific interactions between a host and its parasite. Can. J. Bot. 52, 1339-1347.

Segel, L. A. 1972 Simplification and scaling. SIAM Rev. 14, $547-571$.

Vanderplank, J. E. 1968 Disease resistance in plants. New York: Academic Press.

Received 10 August 1993; accepted 7 September 1993 